\title{
CAD KERNEL AND GRID GENERATION ALGORITHMIC DIFFERENTIATION FOR TURBOMACHINERY ADJOINT OPTIMIZATION
}

\author{
Ismael Sanchez Torreguitart ${ }^{1}$, Tom Verstraete ${ }^{2}$, and Lasse Mueller ${ }^{1}$ \\ ${ }^{1}$ Von Karman Institute for Fluid Dynamics (VKI) \\ Chausse de Waterloo 72, 1640 Rhode-Saint-Gense, Belgium \\ \{ismael.sanchez.torreguitart, lasse.mueller\}@ vki.ac.be \\ ${ }^{2}$ Queen Mary University of London \\ Mile End Road, E1 4NS, London, UK \\ t.verstraete@qmul.ac.uk
}

Keywords: CAD, grid, sensitivities, algorithmic differentiation, adjoint optimization

\begin{abstract}
Although adjoint-based optimization methods have numerous advantages such as the efficient computation of the gradient virtually independent of the number of design variables, the methods are not yet picked up largely by industry. One major bottleneck herein is that adjoint methods mainly work on deforming the CFD grid and as such loose the connection to $C A D$, the industry adopted standard for the design of components. After the optimization a step is required which transforms the optimal shape, defined by grid points, back to a smooth CAD shape. This step is complicated, not easily automated and invariably impairs optimality of the shape as CAD systems will only approximate the optimal shape. The work presented herein is a first step to alleviate the problem by expressing the optimization problem through CAD parameters, rather than working directly on the CFD grid. This allows the optimal shape to remain defined within the CAD tool and through the differentiation of the CAD kernel and grid generation tools with algorithmic differentiation $(A D)$ it is possible to obtain the grid sensitivities propagated to the CAD-based parameters. The in-house CAD and grid generation tools have been differentiated in forward mode as a first step. The VKI LS89 axial turbine vane is selected as a demonstrator to test the methodology. The profile is parameterized by 24 design parameters and the geometrical as well as grid sensitivities are obtained for each parameter, showing the influence of each design parameter on the profile and on the CFD grid coordinates. Finally, the sensitivities obtained by $A D$ are compared against second order accurate central finite difference approximations through changing the perturbation step.
\end{abstract}




\section{INTRODUCTION}

Aerodynamic shape optimization using low-cost, efficient and accurate computational methods is key for the aerospace industry, as it provides the opportunity to make significant design improvements at the early stage of the design chain.

For gradient-based optimization strategies it is essential to compute the total gradient of the design chain, which is the derivative or gradient of the cost function with respect to a vector of design variables. This is usually done by calculating the derivatives separately for the various disciplines used in the design chain (e.g. grid generation, Computational Fluid Mechanics, Computational Solid Mechanics, etc.) and then combining them to obtain the total gradient. The derivatives can be obtained using different methods, either analytically, by finite differences or by differentiating the code(s). Adjoint-based optimization methods $[1,2,3]$ have been introduced for more than two decades and have witnessed a tremendous improvement over time. They have become increasingly popular amongst the gradient-based optimization methods for its efficiency to compute the gradients at a cost that is independent of the number of design variables [4]. However, one major bottleneck herein is that adjoint methods mainly work on altering the shape to be optimized by deforming the CFD grid [5] and as such loose the connection to computer aided design (CAD), the industry adopted standard for the design of components. After the optimization, a step is required which transforms the optimal shape, defined by grid points, back to a smooth CAD shape. Many different procedures have been developed to approximate a given set of grid points with NURBS curves or surfaces [6,7]. Despite the fact that these methods can be successfully employed to obtain a smooth CAD shape, the fitting error may invariably impair optimality of the shape as CAD systems will only approximate the optimal shape.

The emphasis of this work is to present a method in which:

1. the optimal shape remains defined within the CAD tool. The optimization problem herein is expressed by CAD parameters that are directly used in defining the CAD geometry by means of Bézier and B-spline curves.

2. the in-house CAD and grid generation tools are automatically differentiated in forward mode to obtain the exact derivatives of the grid coordinates with respect to the CAD-based design parameters. This allows to accurately predict the sensitivities and circumvent the errors introduced by finite differences.

3. the differentiation of the CAD kernel and grid generation tool in forward mode will then serve to validate the sensitivities in reverse mode. The reverse capability would allow enriching the design space by introducing more design parameters without penalising the computational cost.

The work is carried out using a computer aided design and optimization tool for turbomachinery applications (CADO) [8], which has been used so far mainly in gradient free optimization methods, and hence the work presented herein represents the first steps to differentiate the entire CADO code to make it work in gradient based optimisation. The LS89 $[9,10,11,12]$ axial high pressure turbine nozzle guide vane (HPT NGV) is selected as a demonstrator to test the methodology. This profile was designed and optimized at the von Karman Institute for Fluid 
Dynamics (VKI) for a subsonic outlet flow by the inverse method [9]. It was extensively tested at the VKI laboratories for a large range of Reynolds and Mach numbers and this experimental data was then used to improve the in-house CFD prediction capabilities in the late 80s.

In the following sections the approach to construct the geometry, fluid computational grid and to compute the geometrical and grid sensitivities is presented. Also discussed are the sensitivities obtained by $\mathrm{AD}$ and compared against FD second order central derivatives by tuning the step size.

\section{METHODOLOGY}

The design chain process shown in Fig. 1 starts typically from a set of input design parameters that allow the designer to build a CAD model, from which it is necessary to generate a computational fluid grid to perform CFD analysis. After obtaining a flow converged solution by the primal solver, the cost function is evaluated as a post-processing step. The differentiation of the design chain allows to compute the performance sensitivities which are necessary for gradient based optimization algorithms. The following subsections present a description of the methods employed in this work for the CAD and grid generation steps and the calculation of the geometrical and grid sensitivities.
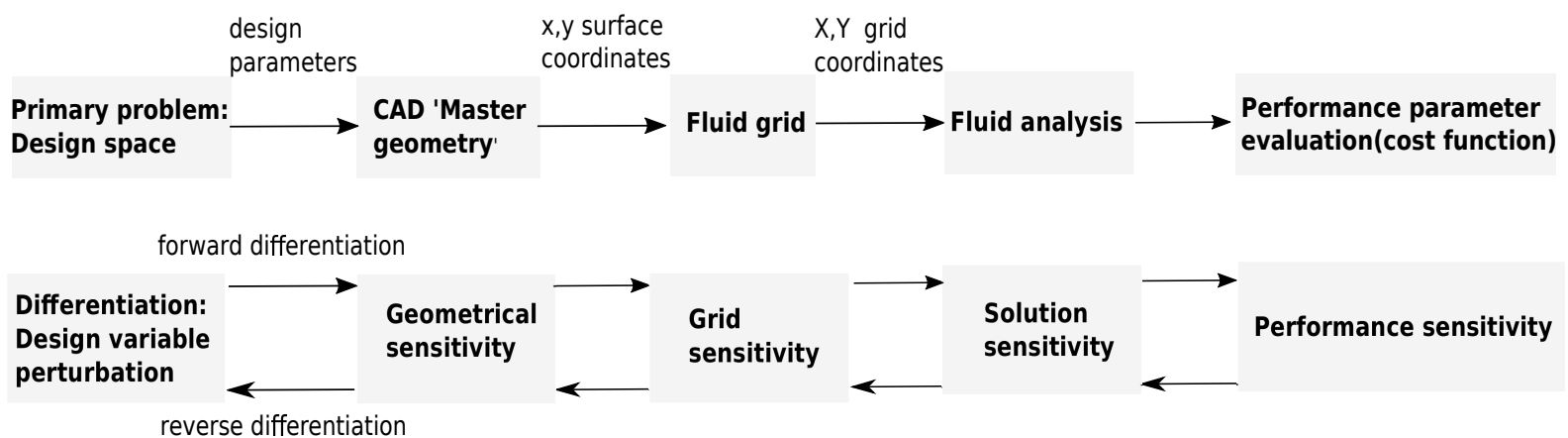

Figure 1: Chain to compute the performance sensitivity

\subsection{Using CAD parameters as design variables}

In gradient based optimization, it is vital to know the performance sensitivities with respect to design parameter changes. Typically, the grid point coordinates have been used as design variables in order to optimize a design [5]. This means that an additional step is required to convert the optimal shape defined by the grid back to a CAD shape. However, it is not guaranteed that the final CAD shape will meet all the manufacturing design requirements and constrains. Furthermore, the generated CAD shape will only approximate the optimal shape given by the CFD grid point cloud [6]. Hence there is an arising need to keep the CAD geometry in the optimization loop.

\subsubsection{Constructing a turbine profile with Bézier and B-spline curves}

The construction of the turbine profile using Bézier curves is based on the parametrization described in [13]. The turbine profile, which is shown in Fig. 2, is defined herein with three independent curves, two B-spline curves for the suction side (SS) and pressure side (PS) and a 
circular arc at the TE to close the profile. In order to construct the profile, first a camber line is constructed, which is used to define the position of the control points of the SS and PS B-spline curves relative to the camber line. The camber line is defined by an inlet blade metal angle, an exit blade metal angle, an axial chord length and a stagger angle. The position of the leading edge and trailing edge is defined by the axial chord length and the stagger angle. By defining an inlet and outlet lines respecting the inlet and outlet blade metal angles, one can intersect the lines and define the point $P_{\text {mid }}$ shown in Fig. 2.

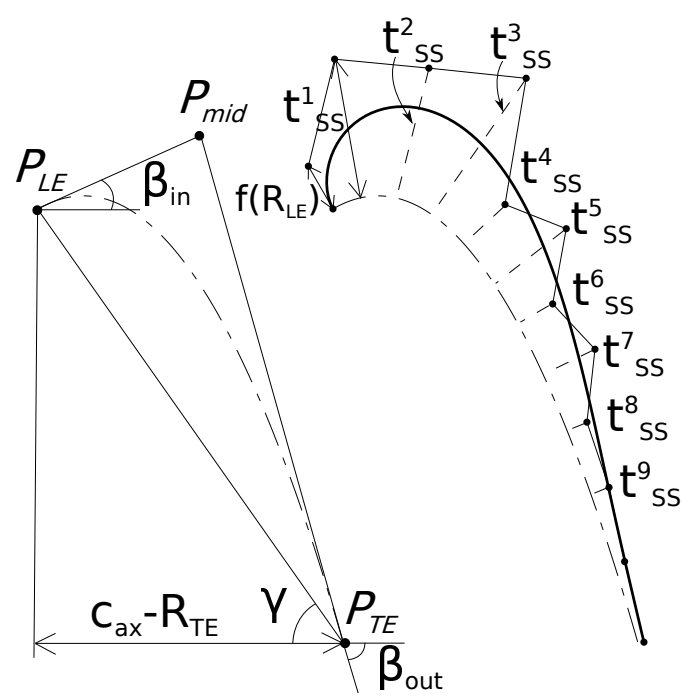

Figure 2: Construction of the suction side by a B-spline curve

The points $P_{L E}, P_{m i d}, P_{T E}$ define the control points of the 2nd order Bézier curve describing the camber line. By specifying a stretch factor and a number of points, the camber line is divided into a number of intervals. For each point obtained on the camber line (except the first and last two points) a normal distance is specified by the designer to obtain the corresponding control point of the SS and PS B-spline curves. The normal distance of the first control point relative to the camber line is a function of the LE radius, in order to guarantee G2 geometric continuity (i.e. equal curvature) between the SS and PS B-splines at the stagnation point of the Leading Edge. The normal distance of the last control point relative to the camber line is equal to the TE radius. For the second last point, the normal distance is computed by specifying a wedge angle. Usually more control points are placed on the SS than on the PS because the highly curved shape of the SS is deemed to play a bigger role than the PS during the aerodynamic shape optimization. Finally, the shape of the cascade is fixed after specifying the pitch, which can be computed for a given solidity. The solidity plays an important role in turbomachinery design practice and this justifies the use of this parameter as an independent variable.

\subsection{Generation of Block-structured smoothed grids}

In the present work a structured grid was preferred as opposed to an unstructured grid to have better accuracy in the flow results. The generation of a boundary conformal curvilinear grid for the turbomachinery flow geometry of this study was achieved by subdividing the domain into multiple structured grid blocks which are independent from each other but share a common interface. A single block approach for a relatively highly staggered profile, such as the LS89 
turbine profile, would result in a poor quality grid due to the high skewness of the cells. Therefore, a multi-block approach was preferred because it allows to have more detailed control of the element size and shape. Figure 3(a) shows the selected multi-block structured topology for the LS89 CFD domain. One O-grid block is placed around the profile and there are six additional $\mathrm{H}$-grid blocks, four of them distributed around the profile and the remaining two being used as the inlet and outlet blocks. The first step in the grid generation process consist of generating

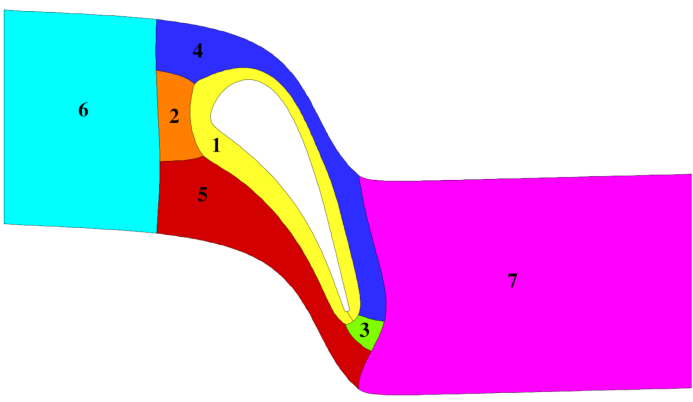

(a) LS89 multi-block structured grid topology

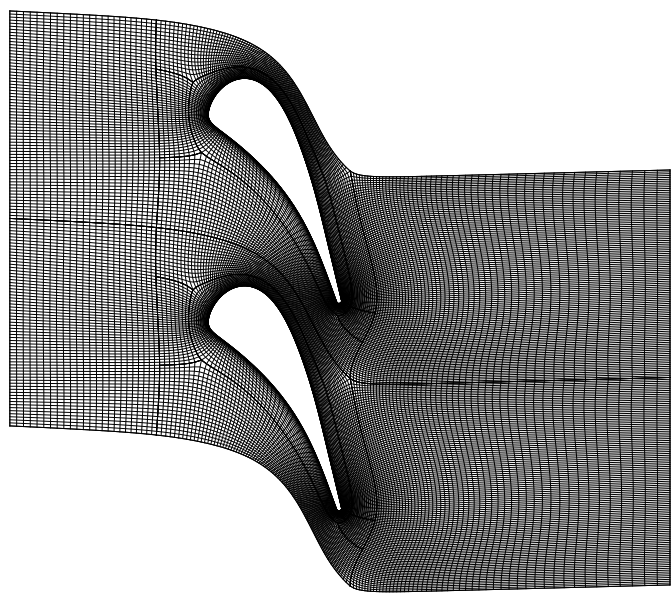

(b) LS89 smoothed multi-block structured grid

Figure 3: LS89 mesh topology and final smoothed grid

an initial grid on the edges of the different blocks. The internal grid is subsequently initialized using Transfinite Interpolation (TFI) equations from the boundary mesh points, which are described by the Eqns. 1 and 2 [14]. Equations 1 and 2 allow to compute the internal grid given the coordinates of the boundaries for $\eta=0,1$ and $\xi=0,1$ (see Fig. 4).

$$
\begin{aligned}
X(\xi, \eta) & =(1-u) x(0, \eta)+u x(1, \eta)+(1-v) x(\xi, 0)+v x(\xi, 1) \\
& -(1-u)(1-v) x(0,0)-u(1-s) x(1,0)-(1-u) v x(0,1)-u v x(1,1) \\
Y(\xi, \eta) & =(1-u) y(0, \eta)+u y(1, \eta)+(1-v) y(\xi, 0)+v y(\xi, 1) \\
& -(1-u)(1-v) y(0,0)-u(1-s) y(1,0)-(1-u) v y(0,1)-u v y(1,1)
\end{aligned}
$$

The parameters $u$ and $v$ from Eqns. 3 and 4 are blending formulas proposed by Soni [15] for the normalised arc-length control functions $s_{1}(\xi), s_{2}(\xi), t_{1}(\eta), t_{2}(\eta)$ along the boundary edges. The normalised arc-length control functions intuitively express the position of each grid point along the edge as a percentage of the total length of the edge. In the present work, $s_{1}(\xi)$ and $s_{2}(\xi)$ are defined along the boundary edges spanning between $t_{1}(\eta=0)$ and $t_{2}(\eta=1)$ respectively, whereas $t_{1}(\eta)$ and $t_{2}(\eta)$ are defined along the boundary edges spanning between $s_{1}(\xi=0)$ and $s_{2}(\xi=1)$.

$$
\begin{aligned}
& u(\xi, \eta)=\frac{\left(1-t_{1}(\eta)\right) s_{1}(\xi)+t_{1}(\eta) s_{2}(\xi)}{1-\left(s_{2}(\xi)-s_{1}(\xi)\right)\left(t_{2}(\eta)-t_{1}(\eta)\right)} \\
& v(\xi, \eta)=\frac{\left(1-s_{1}(\xi)\right) t_{1}(\eta)+s_{1}(\xi) t_{2}(\eta)}{1-\left(t_{2}(\eta)-t_{1}(\eta)\right)\left(s_{2}(\xi)-s_{1}(\xi)\right)}
\end{aligned}
$$




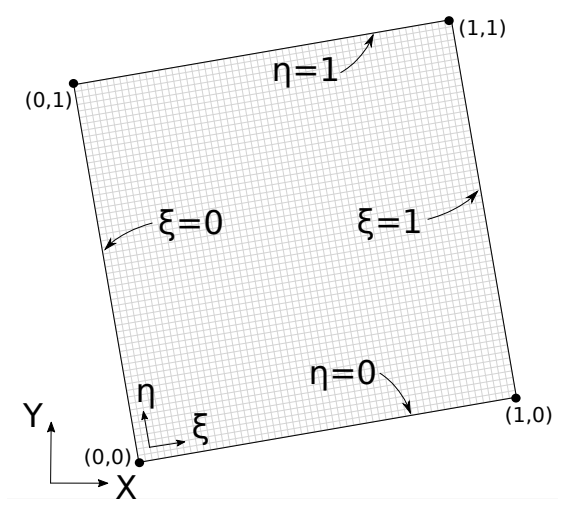

Figure 4: Two dimensional block

The generation of an algebraic grid for each block is efficient and easy to implement but there is no guarantee that the grid lines will not intersect, fold, or that the corners from the side will not be propagated inside the domain. Given the difficulty to generate successful smooth grids using TFI equations, it is convenient to smooth them afterwards by solving a partial differential equation (PDE) of some type (elliptic, hyperbolic, parabolic). The elliptic PDE is commonly used for grid generation for ducted flows. The Elliptic grid generation equations shown in Eqns. 5-6 were pioneered by Thompson et. al. [16] and are employed in this work.

$$
\begin{aligned}
& \xi_{x x}+\xi_{y y}=P(\xi, \eta) \\
& \eta_{x x}+\eta_{y y}=Q(\xi, \eta)
\end{aligned}
$$

These equations, which are also referred to as the Poisson equations, are formulated in the bodyfitted non-orthogonal physical space XY and need to be transformed to the uniform orthogonal computational space $\xi \eta$ in order to solve them efficiently. The inverse transformed system of equations then becomes [14]:

$$
\begin{aligned}
\alpha x_{\xi \xi}-2 \beta x_{\xi \eta}+\gamma x_{\eta \eta} & =-I^{2}\left(P x_{\xi}+Q x_{\eta}\right) \\
\alpha y_{\xi \xi}-2 \beta y_{\xi \eta}+\gamma y_{\eta \eta} & =-I^{2}\left(P y_{\xi}+Q y_{\eta}\right)
\end{aligned}
$$

In Eqns. $7-8$, the greek symbols $\alpha, \beta, \gamma$ represent the scale metric factors of the coordinate transformation and they are defined by $\alpha=x_{\eta}^{2}+y_{\eta}^{2}, \beta=x_{\xi} x_{\eta}+y_{\xi} y_{\eta}, \gamma=x_{\xi}^{2}+y_{\xi}^{2}$. The inverse of the Jacobian is given by $I=\frac{1}{J}=x_{\xi} y_{\eta}-x_{\eta} y_{\xi}$. The system of equations in the transformed space can be approximated using Finite Differences and then solved by means of a standard GS-(S)SOR (Gauss-Seidel Symmetric Successive Over-relaxation) approach.

The $\mathrm{P}$ and $\mathrm{Q}$ source terms are used to control the geometrical aspects of the cells. Grid behaviour control is achieved through the introduction of forcing function terms in the manner of Steger and Sorenson [17] in the O-grid block. The Steger and Sorenson method is an iterative forcing function approach that adjusts the boundary forcing functions to meet a prescribed angle and spacing constraint at the boundary. The forcing function is then interpolated into the domain based on the boundary values of the source terms. In all the H-grid blocks, the source terms have been set to zero and hence the elliptical equations being solved are the so called Laplace equations. 


\subsection{Algorithmic Differentiation}

Algorithmic Differentiation (AD) is a technique for computing analytic derivatives of programs [18]. The idea of AD is to differentiate analytically each elementary mathematical operation performed by the code and accumulating the derivatives by applying the chain rule in an automatic fashion. In this way, it is possible to build up the cost function or derivative of the output with respect to the input variable, which can then be used for an optimization algorithm.

One distinguishes between the forward and reverse mode of AD. In forward mode one is interested to know the derivative of the outputs depending on one setting of the input sensitivities, whereas in reverse mode one is interested in the derivative of all inputs for one single output value. Both modes allows to accurately compute the sensitivities with the best possible machine accuracy. To compute the derivatives for all input parameters in the forward mode it is necessary to run as many simulations as the number of input parameters. Conversely, in the reverse mode you need as many runs as the number of output variables. AD methods can be implemented by either source to source transformation or operator overloading. The former one consists in transferring the primal evaluation trace into a differentiated evaluation trace. The second is based on operator and function overloading. For a more detailed description of the different techniques for evaluating derivatives using AD the reader is referred to [18].

In this work, all the sensitivities have been calculated using ADOL-C [19], an AD tool developed at the Department of Mathematics at the University of Paderborn that is written in $\mathrm{C}++$ and uses overloaded operators and functions. The ADOL-C derivative evaluation routines can be called from C, C++, Fortran and any other language that can be linked with C [19]. Both the geometrical and grid sensitivities have been calculated in tapeless forward mode, which means that the operation count of the added lines necessary to evaluate the sensitivities is a small multiple of that for the underlying code to evaluate the output variable.

\section{APPLICATION}

The LS89 axial turbine aerofoil designed at the Von Karman Institute for Fluid Dynamics is selected to test the method presented in this paper as a first demonstrator. The LS89 is

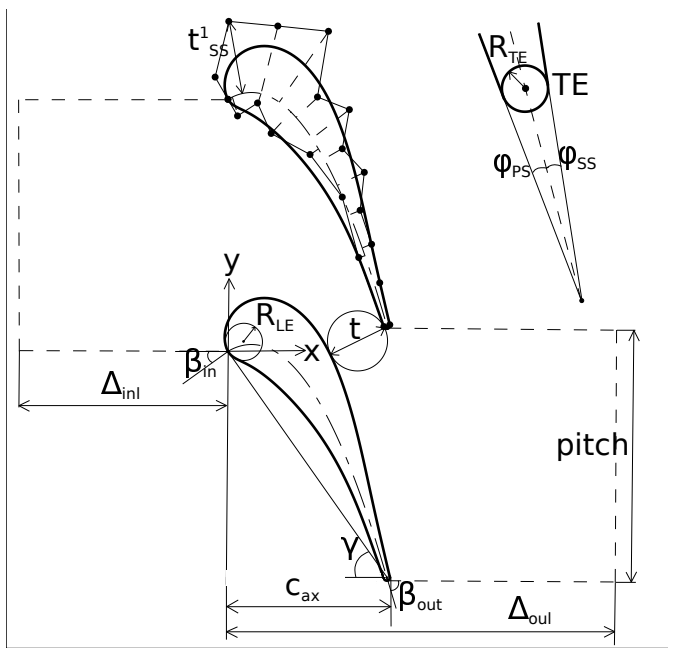

Figure 5: LS89 profile geometry 
a relatively modern high pressure turbine nozzle guide vane (HPT NGV) profile specifically designed for subsonic flows downstream of an aero-engine combustion chamber.

\begin{tabular}{ccc}
\hline Design parameters & Acronyms & Value \\
\hline Solidity & $\sigma$ & 1.118 \\
Axial Chord Length & $c_{a x}$ & $36.955 \mathrm{~mm}$ \\
Stagger Angle & $\gamma$ & $54.9^{\circ}$ \\
LE Radius & $R_{L E}$ & $4.126 \mathrm{~mm}$ \\
TE Radius & $R_{T E}$ & $0.710 \mathrm{~mm}$ \\
TE Wedge Angle SS & $\varphi_{S S}$ & $4.0^{\circ}$ \\
TE Wedge Angle PS & $\varphi_{P S}$ & $2.5^{\circ}$ \\
Inlet Angle & $\beta_{i n}$ & $0.0^{\circ}$ \\
Outlet Angle & $\beta_{\text {out }}$ & $74.0^{\circ}$ \\
Stretching Factors PS & $k_{P S}$ & 1.175 \\
PS Thickness (x4) & $t_{P S}^{1}, \ldots, t_{P S}^{4}$ & see Tab. 2 \\
Stretching Factors SS & $k_{S S}$ & 1.1 \\
SS Thickness (x9) & $t_{S S}^{1}, \ldots, t_{S S}^{9}$ & see Tab. 2 \\
\hline
\end{tabular}

Table 1: Design parameters used to define the LS89 blade profile

\begin{tabular}{ccc}
\hline Bézier Control Point Index j & $t_{S S}^{j}[\mathrm{~mm}]$ & $t_{P S}^{j}[\mathrm{~mm}]$ \\
\hline 1 & 16.750 & 2.250 \\
2 & 15.900 & 6.650 \\
3 & 19.690 & 2.300 \\
4 & 6.750 & 0.040 \\
5 & 10.750 & \\
6 & 4.750 & \\
7 & 6.850 & \\
8 & 2.565 & \\
9 & 2.295 & \\
\hline
\end{tabular}

Table 2: Bézier control point normal distances relative to the camber line

The profile was designed and optimized in the two-dimensional space at the VKI for a 0.9 downstream isentropic Mach number by an inverse method described in [9]. It was tested at the VKI laboratories with the aim to use the experimental data as a benchmark to validate the VKI CFD prediction capabilities in the late 80 s. The complete experimental results were published during the 1990 International Gas Turbine Conference held in Brussels [10]. The final report is the VKI Technical Note TN174 [11]. The LS89 has been tested for different Reynolds and Mach numbers in the VKI-CT2 test facility. A description of different set ups for optimization studies can be found in [12].

The LS89 profile geometry shown in Fig. 5 comes first from point data [11] that has been fitted with the parametrization method described before. The geometry is defined using 24 geometrical parameters shown in Tab. 1 and 2. The position of the Bézier control points of the suction side and pressure side is shown in Fig. 5. 


\section{RESULTS AND DISCUSSION}

The geometrical and the grid sensitivities are obtained for all the design parameters, showing the influence that each design parameter has on the aerofoil surface $X-Y$ coordinates and the CFD grid X-Y coordinates respectively. The discussion herein is limited to the parameters $t_{S S}^{1}, R_{L E}, c_{a x}$ (see Tab. 1) for the sake of simplicity. In the following subsections, the AD geometrical sensitivities, which are defined as the change in the X-Y turbine profile coordinates with respect to a design parameter change, will be discussed first. The grid sensitivities, which are defined as the change in the $\mathrm{X}$-Y grid coordinates with respect to a design parameter change will be discussed next. Finally, the sensitivities obtained by AD will be compared against FD.

\subsection{AD geometrical sensitivities}

Figures 6(a), 6(b) and 6(c) show the magnitude and direction of the geometrical sensitivities obtained for the $t_{S S}^{1}, R_{L E}, c_{a x}$ design parameters, respectively. Different scale factors are used to display the length of the vectors. Figure 6(a) clearly shows that an infinitesimally small

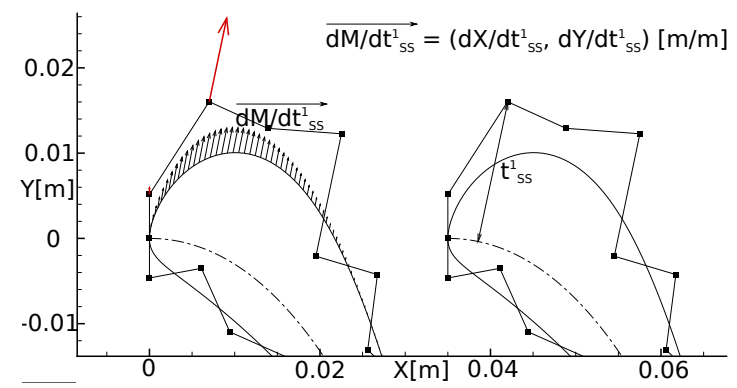

(a) $t_{S S}^{1}$, Vector Scale Factor $=0.01$

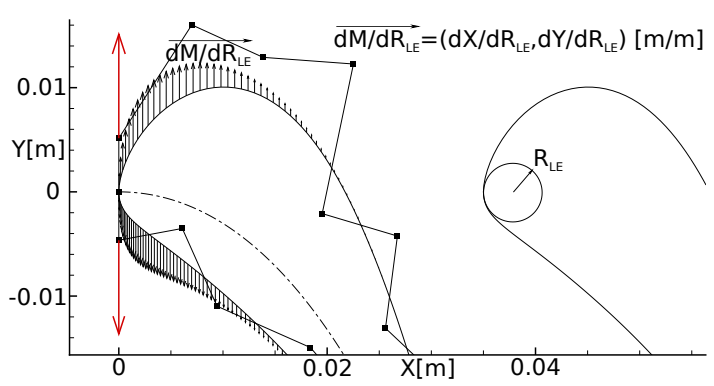

(b) $R_{L E}$, Vector Scale Factor $=0.0016$

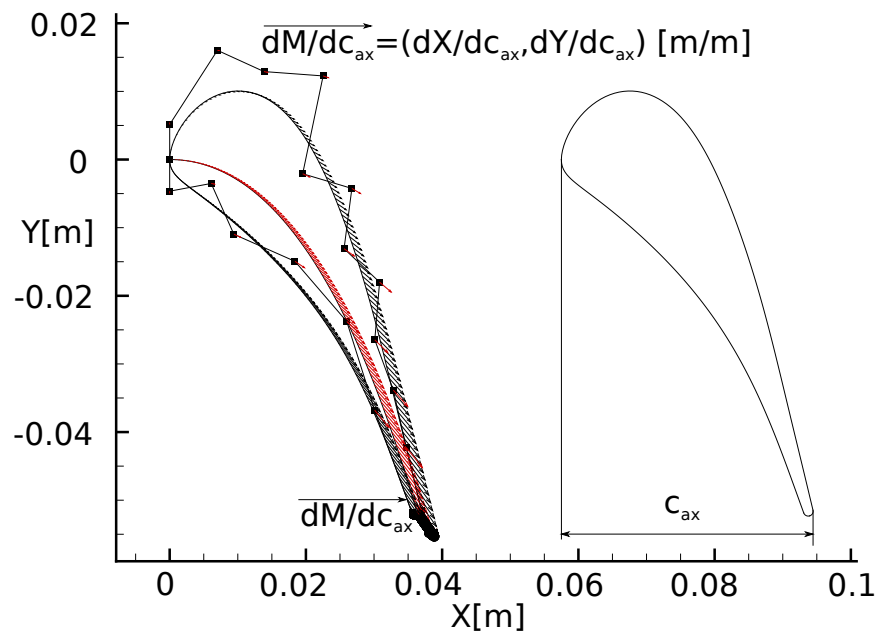

(c) $c_{a x}$, Vector Scale Factor $=0.0025$

Figure 6: AD geometrical sensitivities

perturbation of $t_{S S}^{1}$ causes the movement of the points along the suction side shoulder in the normal direction in which the suction side first Bézier control point position is specified relative to the camber line. A similar perturbation in the $R_{L E}$ forces the first control point of the suction and pressure side to move away from the camber line because their position is a function of the LE radius, making the points along the LE region to move in the y direction accordingly 
as displayed in Fig. 6(b). Figure 6(c) shows that a perturbation in the $c_{a x}$ causes the points along the camber line, SS and PS curves to move away from the stagnation point at the LE. Although the scale factor does not allow to show the magnitude of all the sensitivities, all the control points (except the first of the SS and PS curves) sensitivity vectors point away from the stagnation point because the TE point ( $P_{T E}$ from Fig. 2 ) also moves away in both $\mathrm{x}$ and $\mathrm{y}$ directions. The TE point movement is not in the $\mathrm{x}$ direction only because an increase of axial chord by virtue of a constant stagger angle requires the TE point (and the camber line) to move in the $(\mathrm{x}, \mathrm{y})$ space.

\subsection{AD grid sensitivities}

Figures 7(a), 7(b) and 7(c) show the magnitude of the grid sensitivities obtained for the $t_{S S}^{1}$, $R_{L E}, c_{a x}$ design parameters, respectively. In general, a perturbation in the design parameter can cause two type of perturbations in the CFD grid point coordinates. First, the grid point distribution along the edge of the curve itself can change due to the fact that the normalised arc-length functions used to distribute points in the edge undergo some changes. This type of perturbation that moves the surface grid points tangentially to the surface can also propagate into the interior domain of the grid but it has a tendency to decay rapidly. Secondly, the grid points coordinates in the fluid domain change as well according to the overall grid point field movement sensed by them. The source of this perturbation is stronger than for the first type and originates in the region where the geometrical sensitivities are not zero. Figure 7(a) clearly shows that a small perturbation in the $t_{S S}^{1}$ causes the grid points in the fluid domain above the SS shoulder to move and this movement also propagates into the PS fluid domain through the periodic interface. The rear suction side grid points are also affected due to the fact that the arclength distribution changes. The geometrical throat is used herein to split the SS curve into two edges to have more control of the grid point distribution along the SS curve. This means that the geometrical throat on the SS (see Fig. 7(a)) is the end of the SS pre-throat and the beginning of the SS post-throat edges, which are using different arc-length distributions. Therefore, it is expected that a perturbation in SSThck1 causes a grid point position variation along the SS pre-throat and post-throat edges because the length of these edges changes. However, as the geometrical throat X-Y coordinates remain fixed, the grid points in the vicinity of the SS geometrical throat do not sense any perturbation.

Figure 7(b) shows that the biggest influence of $R_{L E}$ is in the vicinity of the largest geometrical sensitivities (see Fig. 6(b)). The perturbation on the SS also propagates into the PS interior domain through the periodic interface. Also, the changes in the normalised arc-length functions for the PS and SS edges result into grid point movements in both PS and SS curves. As the SS is more curved than the PS, it is expected that a perturbation in the $R_{L E}$ changes the total length of the SS curve more than for the PS. This is the reason why the $R_{L E}$ grid sensitivities at the rear SS are bigger than the rear PS. The grid point displacements along the SS and PS also propagate into the interior of the fluid domain but decay more rapidly than in those that propagate from the LE region. The minimum sensitivity areas around the profile correspond to those grid points that are fixed, like the stagnation point, the geometrical throat position, and the SS and PS end points towards the TE. In these points, the arc-length functions do not change because they take the values zero or one, depending the orientation in which the grid point distribution has been applied to the edges.

Figure 7(c) shows a non-periodic character, for the displacement field is not repeated passage after passage. This is because the solidity, as an independent parameter, remains constant under a change of the axial chord length, resulting is a proportional change in pitch. Identical points 


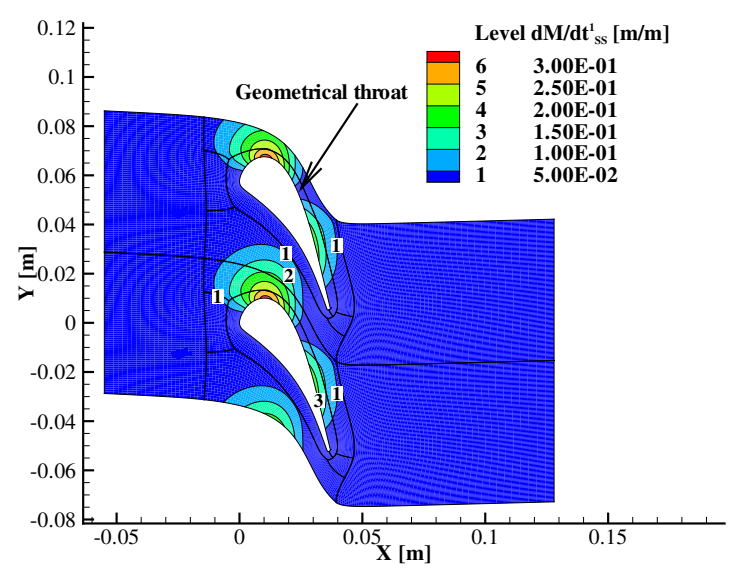

(a) $t_{S S}^{1} \mathrm{AD}$ grid sensitivities

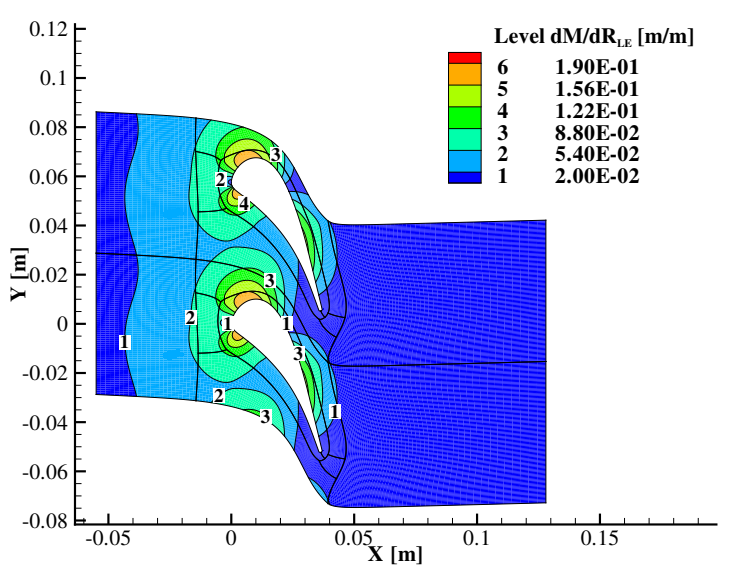

(b) $R_{L E}$ grid sensitivities

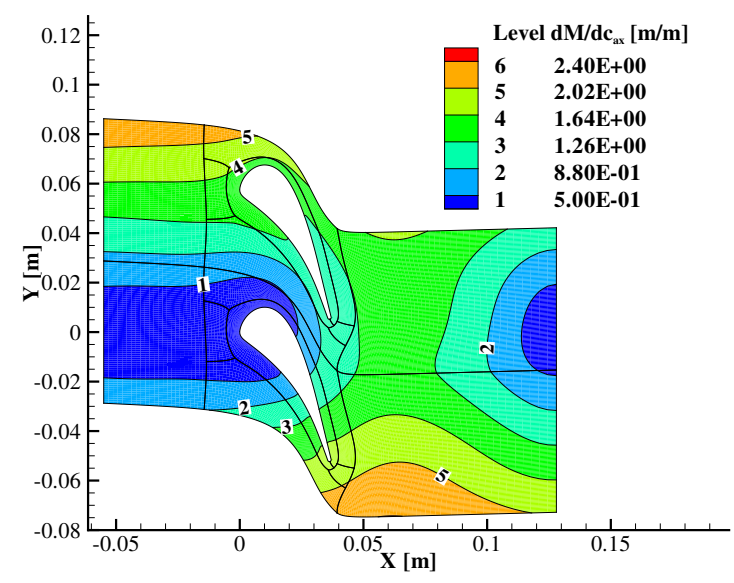

(c) $c_{a x}$ grid sensitivities

Figure 7: AD grid sensitivities

on the opposite side from the periodic boundary will experience a jump in the y-component of the grid sensitivity, proportional to the change in pitch. In the figure, the jump value has been added to the top CFD domain such that a continuous field is visualized. The choice to use solidity as an independent parameter is inspired by it's importance in classical turbomachinery design practice.

\subsection{AD vs FD comparison}

The geometrical and grid sensitivities obtained by AD are compared against FD second order central derivatives by tuning the step size. Figures 8(a) and 8(b) show the maximum error in magnitude between the FD approximated and the exact $\mathrm{AD}$ sensitivities for the $t_{S S}^{1}, R_{L E}$ and $c_{a x}$ design parameters. The error is very sensitive to the chosen step size and the step size needs to be relatively small to have a small error. Figures 8(a) and 8(b) show that the error in both cases reduces with order two as the step size is reduced up to a critical step size, as to be expected for a central second order FD scheme. If the step size is chosen too small below this critical step size, the numerical representation of the FD approximation becomes unstable and more error-prone due to the limited machine accuracy. The error increases with order one approximately if the step size is reduced below the critical step size. Figures 9(a), 9(b) and 9(c) show the grid sensitivity error for the $t_{S S}^{1}, R_{L E}$ and $c_{a x}$ design parameters if the step size 
is $1 \mathrm{E}-03$ [m]. It has to be noted that the maximum grid sensitivity error shown in Fig. 9(b) is relatively large, as it is only two orders of magnitude lower than the maximum grid sensitivities shown in Fig. 7(b), and will eventually result in wrong gradient information.

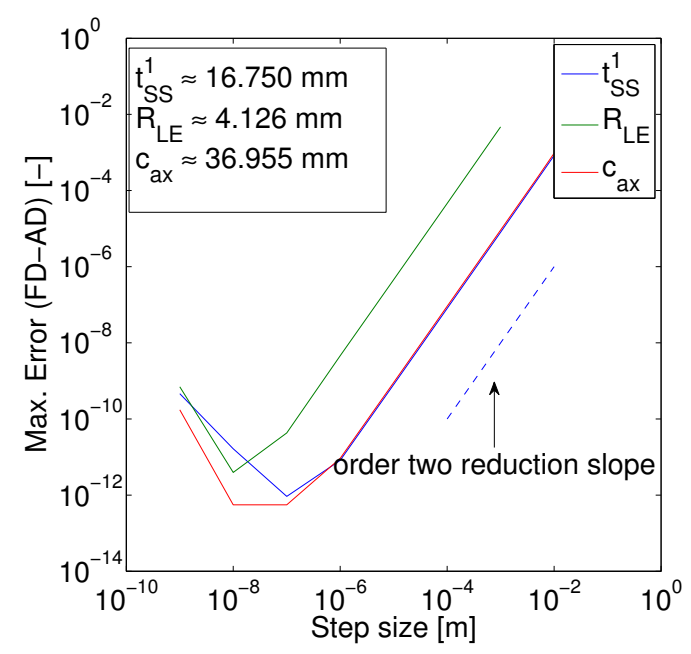

(a) Geometrical sensitivity error

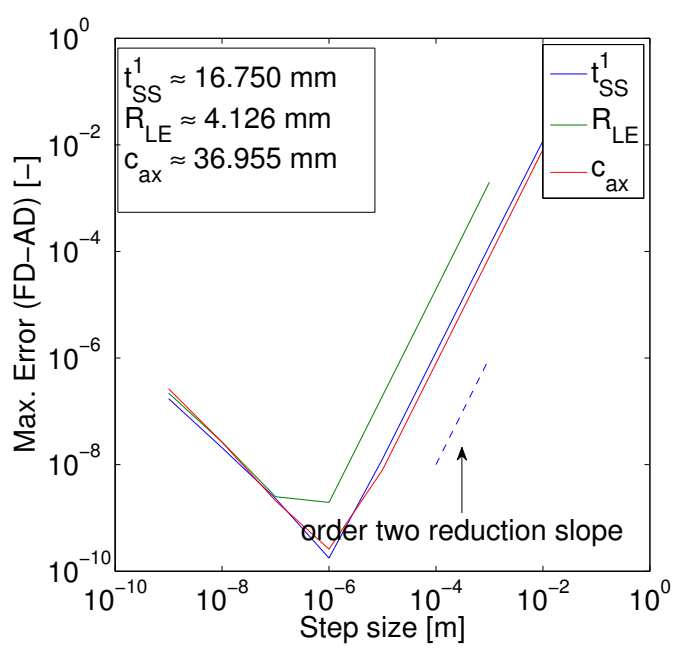

(b) Grid sensitivity error

Figure 8: Max Error between the FD and AD

\section{CONCLUSIONS}

The method presented herein allows to integrate the CAD kernel and grid generation tools within the CFD adjoint-based optimization algorithm by expressing the optimization problem through CAD parameters, rather than working directly on the CFD grid. This allows the optimal shape to remain defined within the CAD tool and to differentiate the CAD kernel and grid generation tool with algorithmic differentiation (AD) to obtain the grid sensitivities propagated to the CAD-based parameters. The in-house CAD and grid generation tools have been differentiated in forward mode and tested in the VKI LS89 axial high pressure turbine nozzle guide vane. The two dimensional profile, constructed by means of Bézier and B-spline curves, is parametrized by 24 design parameters and the geometrical as well as grid sensitivities are obtained for each, showing the influence that each design parameter has on the profile and on the CFD grid coordinates. Finally, the sensitivities obtained by AD are compared against finite differences second order central derivatives by tuning the step size. The error introduced by FD is very sensitive to the chosen step size and its magnitude can be relatively close to the sensitivity value itself if the step size is not chosen appropriately. The algorithmic differentiation of the cad kernel and grid generation tool is a promising approach to obtain accurate sensitivities with the best machine accuracy. Although in the present approach the AD sensitivities are obtained in forward mode mainly, algorithmic differentiation also offers the reverse capability. Further work will focus in differentiating the CAD kernel and grid generation tool in reverse mode. The sensitivities computed in forward mode will serve to validate those obtained by the reverse mode. The differentiated code will be integrated afterwards with the CFD solver and the gradient-based optimization algorithm. Finally, the method will be extended to more complicated 3D turbomachinery test cases. 


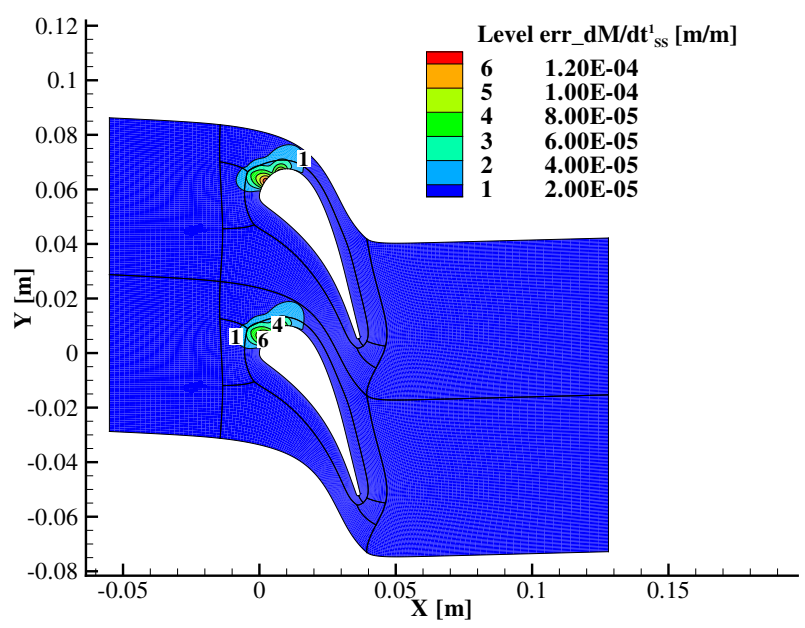

(a) $t_{S S}^{1}$ grid sensitivity error, step size $=1 \mathrm{E}-03$

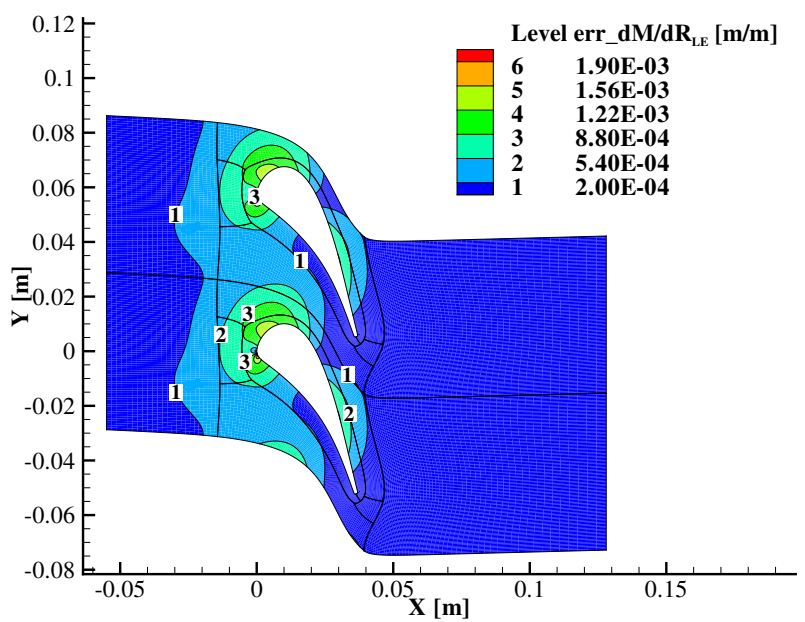

(b) $R_{L E}$ grid sensitivity error, step size $=1 \mathrm{E}-03$

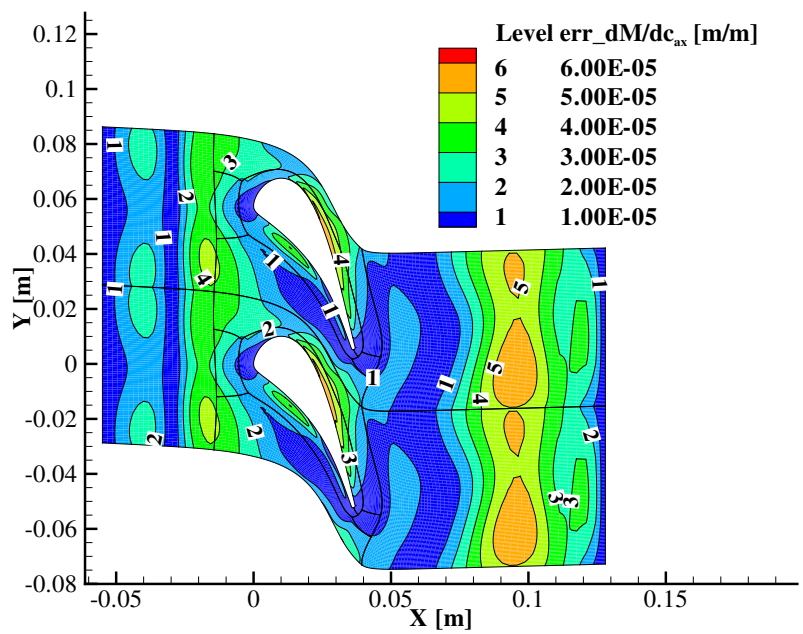

(c) AxCh grid sensitivity error, step size $=1 \mathrm{E}-03$

Figure 9: Grid sensitivity error for step size $=1 \mathrm{E}-03$ 


\section{REFERENCES}

[1] Jameson, A., "Aerodynamic design via control theory," Journal of scientific computing, Vol. 3, No. 3, 1988, pp. 233-260.

[2] Pironneau, O., "On optimum design in fluid mechanics," Journal of Fluid Mechanics, Vol. 64, No. 01, 1974, pp. 97-110.

[3] Giles, M. B. and Pierce, N. A., "An introduction to the adjoint approach to design," Flow, turbulence and combustion, Vol. 65, No. 3-4, 2000, pp. 393-415.

[4] Peter, J. E. and Dwight, R. P., "Numerical sensitivity analysis for aerodynamic optimization: A survey of approaches," Computers \& Fluids, Vol. 39, No. 3, 2010, pp. 373-391.

[5] Jameson, A., "Efficient aerodynamic shape optimization," AIAA paper, Vol. 4369, 2004, pp. 2004.

[6] Becker, G., Schäfer, M., and Jameson, A., "An advanced NURBS fitting procedure for post-processing of grid-based shape optimizations," 49th AIAA Aerospace Sciences Meeting, Orlando, FL, USA, 2011.

[7] Leal, N., Leal, E., and Branch, J. W., "Simple method for constructing nurbs surfaces from unorganized points," Proceedings of the 19th international meshing roundtable, Springer, 2010, pp. 161-175.

[8] Verstraete, T., "Cado: A computer aided design and optimization tool for turbomachinery applications," 2nd Int. Conf. on Engineering Optimization, Lisbon, Portugal, September, 2010, pp. 6-9.

[9] Van den Braembussche, R., Léonard, O., and Nekmouche, L., "Subsonic and transonic blade design by means of analysis codes," Computational Methods for Aerodynamic Design (Inverse) and Optimization, AGARD CP, Vol. 463, 1990.

[10] Arts, T. and De Rouvroit, M. L., "Aero-Thermal Performance of a Two Dimensional Highly Loaded Transonic Turbine Nozzle Guide Vane: A Test Case for Inviscid and Viscous Flow Computations," ASME 1990 International Gas Turbine and Aeroengine Congress and Exposition, American Society of Mechanical Engineers, 1990, pp. V001T01A106-V001T01A106.

[11] Arts, T., Lambertderouvroit, M., and Rutherford, A., "Aero-thermal investigation of a highly loaded transonic linear turbine guide vane cascade. A test case for inviscid and viscous flow computations," NASA STI/Recon Technical Report N, Vol. 91, 1990, pp. 23437.

[12] Mueller, L. and Verstraete, T., The LS89 Optimization Test Case, 2016, Available at http://aboutflow.sems.qmul.ac.uk/content/events/munich2016/ benchmark/testcase2/files/LS89TestCase.pdf, url date 2016-02-03.

[13] Pierret, S., Designing turbomachinery blades by means of the function approximation concept based on artificial neural network, genetic algorithm, and the navier-stokes equations, Ph.D. thesis, Von Karman Institute for Fluid Dynamics, 121999. 
[14] Thompson, J. F., Soni, B. K., and Weatherill, N. P., Handbook of grid generation, CRC press, 1998.

[15] SONI, B., "Two-and three-dimensional grid generation for internal flow applications of computational fluid dynamics," 7th Computational Physics Conference, 1985, p. 1526.

[16] Thompson, J. F., Thames, F. C., and Mastin, C. W., "Automatic numerical generation of body-fitted curvilinear coordinate system for field containing any number of arbitrary twodimensional bodies," Journal of computational physics, Vol. 15, No. 3, 1974, pp. 299-319.

[17] Steger, J. and Sorenson, R., "Automatic mesh-point clustering near a boundary in grid generation with elliptic partial differential equations," Journal of computational physics, Vol. 33, No. 3, 1979, pp. 405-410.

[18] Griewank, A. and Walther, A., Evaluating derivatives: principles and techniques of algorithmic differentiation, Siam, 2008.

[19] Walther, A. and Griewank, A., "A Package for the Automatic Differentiation of Algorithms Written in C/C+," URL https://projects. coin-or. org/ADOL-C, 2014.

[20] Piegl, L. and Tiller, W., The NURBS book, Springer Science \& Business Media, 2012. 